\title{
Festl, Michael G. (2019) Scheitern an Kontingenz. Politisches Denken in der Weimarer Republik
}

\author{
Frankfurt/New York: Campus. 457 Seiten. 45,00€
}

\author{
Marcus Llanque
}

Angenommen: 21. Dezember 2020 / Online publiziert: 8. Januar 2021

(C) Der/die Autor(en) 2021

Die an der Universität St. Gallen entstandene Druckfassung einer Habilitationsschrift versucht anhand des Begriffs der Kontingenz, das politische Denken während der Weimarer Republik neu zu sortieren. Anhand von fünf Modellen der Kontingenz, die jeweils am Beispiel zweier Autoren dargestellt werden, will Michael G. Festl sich einem Modell nähern, dessen Umgang mit Kontingenz ,demokratischen Verhältnissen förderlich“" ist (S. 19). Beginnend mit der am weitesten die Kontingenz ablehnenden Position steht das „Leugnungsmodell der Kontingenz“, das am Beispiel von Oswald Spengler und Georg Lukács demonstriert wird, gefolgt vom „Aufdeckungsmodell der Kontingenz“ (Stefan George und Ernst Kantorowicz einerseits und Moeller van den Bruck andererseits), dem „Produktivitätsmodell der Kontingenz“ (Ernst Jünger und Walter Benjamin), dem „Entscheidungsmodell der Kontingenz“ (Ernst Robert Curtius und Carl Schmitt) sowie dem „Dynamikmodell der Kontingenz“ (Hans Kelsen und Rabindranath Tagore), das sich der Kontingenz gegenüber am weitesten aufgeschlossen zeigt.

Die dem philosophischen Wissenschaftsdiskurs zugehörige Arbeit stimmt von der Anlage her zunächst hoffnungsfroh. Hier werden nicht pauschal Ideologien einander gegenübergestellt (etwa mit dem Liberalismus als kontingenzfreundlichem Denkmuster) und es werden auch Autoren gewählt, die nicht als Theoretiker bekannt sind, sondern eher repräsentativ für bestimmte Denkweisen stehen (z. B. Kantorowicz, Jünger, Curtius). Warum gleichsam an der Spitze des kontingenzfreundlichen Denkens Tagore steht, und dies in einer Arbeit zu Weimar, ist eine ungewöhnliche Setzung, die nicht durch die bloße Behauptung überzeugen kann, Tagore sei fester Bestandteil der „kulturellen und philosophischen Szenerie“ (S. 373) der deutschen

M. Llanque ( $\triangle)$

Professur für Politische Theorie, Universität Augsburg, Augsburg, Deutschland

E-Mail: marcus.llanque@phil.uni-augsburg.de 
Zwischenkriegszeit gewesen. Jedenfalls ist die Auswahl der Autoren erfrischend und zeigt das denkerische Potenzial dieser Epoche.

Der Erste Weltkrieg habe die Wirkung eines „Kontingenzschocks“ (S. 11) gehabt und damit dem politischen Denken Weimars sozusagen sein Hauptproblem diktiert. Diese Annahme des Bruchs und der durch ihn erfolgten Irritation von Erfahrungsraum und Erwartungshorizont ist eine Annahme, die den Analysen Weimars wie der Zwischenkriegszeit im Allgemeinen zugrunde gelegt wird. Hier sei an Norbert Bolz' „Auszug aus der entzauberten Welt“ von 1989 erinnert. Wenn der Weltkrieg als Kontingenzschock beschrieben wird, hätte man vermuten können, dass die weiteren historischen Ereignisse, welche diese Qualität hatten und den Weimarer Diskurs strukturierten, berücksichtigt werden. Auch die Revolution war ein Kontingenzschock mit der plötzlich vorhandenen Möglichkeit, sich politisch in eine völlig andere Richtung zu bewegen als nur die Tradition zu reformieren; schockierend war die erste Inflation von 1923 mit ihrer Erschütterung der sozialen Verankerung des Bürgertums; den intensivsten Kontingenzschock erzeugte die zweite Inflation von 1929, die Zeitgenossen nur eine wenig tröstliche Erklärung bot für den völlig überraschenden Wahlsieg der Nazis 1930, für sich betrachtet auch ein Schock. Das weitere Erleben der Kontingenz nach dem Weltkrieg wird vom Verfasser jedoch nicht behandelt. Hier zeigt sich das methodische Problem der gewählten Anordnung: Modelltheoretisch vorzugehen, vernachlässigt zwangsläufig die Genese dieser Theorien im Kontext des Ablaufs der kontroversen Theoriediskussionen.

Was ist nun das erkenntniserweiternde Potenzial des Kontingenz-Begriffs, der von Festl in den Mittelpunkt gestellt wird? Die „Kontingenz“ ist ein seit Jahren immer intensiver diskutierter, in der Demokratietheorie immer zentraler verwendeter Begriff, weshalb man gespannt sein durfte, welche Aufklärungsleistung hier die ,prima scientia“ erbringen würde. Aber von Kontingenz selbst ist zwar viel die Rede, sie bleibt aber nur vage begrifflich erörtert, selten finden sich mehr als lapidare Aussagen, wonach es sich um einen negativen Begriff einer Auffassung handle, der zufolge gilt: ,,[E]s gibt keine feste Ordnung der Dinge, keine Notwendigkeiten des gesellschaftlichen Lebens, keine determinierte Geschichte etc.“ (S. 13). Die Ideengeschichte der Kontingenz, die Peter Vogt 2014 vorlegte, findet sich im Literaturverzeichnis, ihre begrifflichen Leistungen werden aber nicht zurate gezogen. Es ist aufschlussreich, wie vom Verfasser die Unterstellung der Kontingenz zur „Tatsache“ (S. 102) der modernen Welt erhoben wird, sodass beispielsweise die theoretischen Bemühungen, etwa geschichtsphilosophisch mit Kontingenz umzugehen, als Leugnung der Kontingenz dechiffriert wird, im Sinne mangelnder „Akzeptanz“ (S. 103) dieser „Tatsache“. Ist es aber schon eine intellektuelle Fehlleistung, welche auf subjektive Probleme des Nichtglaubens schließen lässt, wenn ein Umgang mit Kontingenz gesucht wird, welcher der unterstellten Kontingenz des Geschehens einen Sinn abgewinnen will, um sich in der kontingenten Welt wenigstens zu orientieren?

Ein erheblicher Teil dessen, was vom Verfasser als Problem der Kontingenz thematisiert wird, wurde zeitgenössisch als Relativismusproblem erörtert, und zwar unter einem erkenntnistheoretischen wie wissenschaftstheoretischen Gesichtspunkt. Wir haben bereits interessante Arbeiten wie die von Kari Palonen (,Das ,Webersche Moment‘. Zur Kontingenz des Politischen“, 1998), die bei Max Weber ansetzen. Auch die Politische Theorie von Oliver Marchart mit ihrer postfundamentalisti- 
schen Ausrichtung beschäftigt sich intensiv mit dem Kontingenz-Begriff. Mag sie auch kein Beitrag zum Weimarer Denken sein, so schließt sie an Heideggers Philosophie an, die für viele junge Weimarianer den Bruch mit der alten Metaphysik initiierte. Hier wäre die Positionierung des Verfassers zu der heutigen KontingenzDebatte aufschlussreich gewesen, die in Weimar ihren Ausgang nahm. Heidegger wird aber nur am Rande erwähnt, insbesondere als Hintergrund Jüngers.

Auf welcher Ebene bewegt sich das Kontingenz-Argument? Von welchem Typus an Notwendigkeit ist hier die Rede? Ist ein Denken der Notwendigkeit im metaphysisch-ontologisch-geschichtsphilosophischen Sinne vergleichbar mit dem Denken der Notwendigkeit im Bereich zwischen politischer Handlungsfreiheit und Ordnungserfordernissen? Sind demokratische Gesetze kontingent und daher weniger beachtlich? Die Analyse politischen Denkens mithilfe von Kontingenz, die von sich behaupten muss, nicht selbst kontingent zu sein, sondern als (deterministische) Kategorie dogmatisch fixiert wird, wonach nichts determiniert sein kann, könnte an politischen Problemen der Demokratie vorbeigehen, auf die laut Festl alle Kontingenz notwendig zuläuft.

Das Eigentümliche dieser Weimarer Welt war ja - wie der Verfasser selbst zeigt der Umstand, dass so viele jeweils als notwendig angesehene ideologische und geschichtsphilosophische Positionen vorhanden waren. Das führt dazu, dass die Demokratie selbst ein Problem mit Kontingenz hat, dass sich nicht dadurch auflöst, dass man alles für kontingent erklärt. Denn die Sinnstiftung des kontingenten Geschehens verbleibt, oder man muss zeigen, welches Potenzial an Sinnstiftung dem Glauben an Kontingenz zu entnehmen ist.

Wie wenig mit der kruden Entgegensetzung von Kontingenz und Notwendigkeit gewonnen ist, zeigt sich beispielsweise an der Interpretation von Kelsen, der Festl zufolge einer „Affirmation“ (S. 364) der Kontingenz am weitesten kommt. Hier wird die von Kelsen diskutierte Gegenüberstellung von absoluter und relativer Weltanschauung als Paradebeispiel dafür gesehen, was der Verfasser unter dem Gegensatz von Notwendigkeit und Kontingenz fasst. Was aber Kelsen diskutiert, ist das Problem der demokratischen Offenheit: Die Demokratie, die sich auf das formale Zustandekommen von immer nur vorläufig verbindlichen Normen konzentriert, muss ihrer Logik nach offen für jeden politischen Inhalt sein, was in Konfrontation mit einer Weltanschauung, die von ihren politischen Zielvorstellungen absolut überzeugt und daher nicht zu Kompromissen bereit ist, zu fundamentalen Problemen führen muss. Kelsen hat keine Antwort hierauf, er formuliert zunächst die Problemanzeige. Seine Überlegungen hinsichtlich der Ausformulierung eines demokratischen Ethos (unterstützt durch politische Erziehung) werden von Festl bereits als Abkehr vom reinen Kontingenz-Denken verstanden und kritisiert. So wird das Kontingenz-Denken freilich zum ungewollten Komplizen radikaler, totalitärer Denkweisen, weil die Demokratie nicht nur offengehalten werden soll, sondern wehrlos gemacht wird.

Philosophisch wäre es interessant gewesen, eine kantianisch geprägte Logik von Kontingenz (wie sie bei Kelsen am Werk ist) im Sinne eines Entweder-oder und der Aufspannung eines Dualismus einerseits mit einer hegelianisch geprägten Logik andererseits zu vergleichen, in welcher das Verhältnis von Freiheit und Notwendigkeit dialektisch begriffen wird. Hegels Geschichtsphilosophie, nicht seine Logik, wird als Hintergrund von Jüngers politischem Denken angerissen (dort auch eini- 
ge Passagen zu Heidegger), aber es wäre interessant gewesen, zu sehen, wie etwa Hermann Heller in Kritik zu Schmitt mit dem Verhältnis von Freiheit und Notwendigkeit umging. Auf Heller zu verweisen meint nicht den banalen Hinweis darauf, dass einige interessante Autoren nicht behandelt wurden (was notwendigerweise der Fall ist angesichts der begrenzten Kapazitäten einer Monografie), sondern will auf eine systematische Lücke des Arguments aufmerksam machen.

Funding Open Access funding enabled and organized by Projekt DEAL.

Open Access Dieser Artikel wird unter der Creative Commons Namensnennung 4.0 International Lizenz veröffentlicht, welche die Nutzung, Vervielfältigung, Bearbeitung, Verbreitung und Wiedergabe in jeglichem Medium und Format erlaubt, sofern Sie den/die ursprünglichen Autor(en) und die Quelle ordnungsgemäß nennen, einen Link zur Creative Commons Lizenz beifügen und angeben, ob Änderungen vorgenommen wurden.

Die in diesem Artikel enthaltenen Bilder und sonstiges Drittmaterial unterliegen ebenfalls der genannten Creative Commons Lizenz, sofern sich aus der Abbildungslegende nichts anderes ergibt. Sofern das betreffende Material nicht unter der genannten Creative Commons Lizenz steht und die betreffende Handlung nicht nach gesetzlichen Vorschriften erlaubt ist, ist für die oben aufgeführten Weiterverwendungen des Materials die Einwilligung des jeweiligen Rechteinhabers einzuholen.

Weitere Details zur Lizenz entnehmen Sie bitte der Lizenzinformation auf http://creativecommons.org/ licenses/by/4.0/deed.de. 